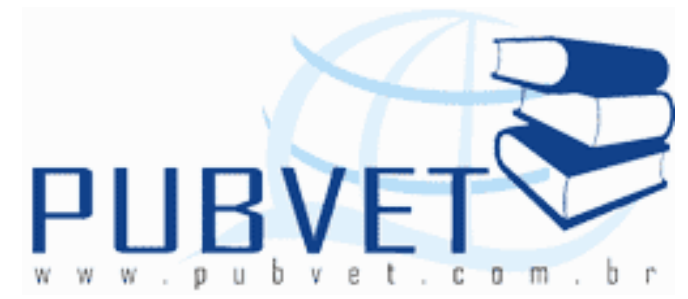

PUBVET, Publicações em Medicina Veterinária e Zootecnia.

Disponível em: <https://doi.org/10.31533/pubvet.v02n11a455>.

\title{
Diagnóstico ultra-sonográfico de dioctofimose em cão - relato de caso
}

Bruna Junca Pereira ${ }^{1}$, Clóvis Holanda2 ${ }^{2}$ Daniel Capucho de Oliveira ${ }^{2}$, Bernardo Fernandes Lopes ${ }^{3}$, Fabiano Séllos Costa ${ }^{4}$

1- Residente em Diagnóstico por Imagem do Centro Universitário Vila Velha ES.

2- Discente do Curso de Medicina Veterinária - Centro de Ciências Agrárias da Universidade Federal do Espírito Santo - ES.

3- Médico Veterinário autônomo, DIAGNOPET, Vitória, ES.

4- Professor Adjunto de Diagnóstico por Imagem e Clínica Médica de Pequenos Animais - Centro de Ciências Agrárias da Universidade Federal do Espírito Santo - ES.

\section{RESUMO}

A dioctofimose é causada pelo Dioctophyma renale e acomete o rim de cães domésticos e selvagens. Seu diagnóstico através do exame clínico, urinálise, radiografia, ultra-sonografia e achados acidentais em cirurgias e necropsias. $\mathrm{O}$ animal relatado apresentava-se com aumento de volume abdominal sendo requisitado um exame ultra-sonográfico. A imagem do rim direito permitiu caracterizar a presença do parasito. Em seguida realizou-se a urinálise, na qual se visualizou ovos do verme. Ressalta-se o incomum achado ultra-sonográfico 
Pereira, B.J., Holanda, C., Oliveira, D.C. et al. Diagnóstico ultra-sonográfico de dioctofimose em cão - relato de caso. PUBVET, Londrina, V. 2, N. 47, Art\#455, Nov4, 2008.

de dioctofimose na rotina de exames ultra-sonográficos, sendo este um método extremamente útil para o diagnóstico desta enfermidade.

Palavras - chave: Dioctophyma renale, ultra-sonografia, cães.

\title{
Ultra-sonographic diagnosis of dioctophymosis in a dog - case report
}

\begin{abstract}
Dioctophymosis is caused by Dioctophyma renale and affects the kidneys of domestic and wild dogs. The diagnosis is made by clinical examination, urinalysis, radiography, ultrasound and incidental findings in surgery and necropsies. The reported animal presented increasing in abdominal volume being requested an ultrasonographic examination. The image of the right kidney allowed the characterization of the parasite's presence. Then, there was realized an urinalysis, in which were visualized the worm's eggs. It is detached the unusual ultrasonographic finding of dioctophymosis in routine ultrasound examinations, being this an extremely useful method for diagnosing this disease.
\end{abstract}

Keywords: Dioctophyma renale, ultrasound, dogs.

\section{INTRODUÇÃO}

O nematóide Dioctophyma renale (Goeze, 1782) é um helminto de coloração avermelhada com tamanho variando conforme o seu hospedeiro, chegando as fêmeas a atingir $100 \mathrm{~cm}$ de comprimento por 5 a $12 \mathrm{~mm}$, sendo conhecido também como "verme gigante do rim" [1]. Alimentam-se de tecidos, sangue, secreções e muco [5]. O parasito destrói o tecido renal podendo causar peritonite [4]. 
Pereira, B.J., Holanda, C., Oliveira, D.C. et al. Diagnóstico ultra-sonográfico de dioctofimose em cão - relato de caso. PUBVET, Londrina, V. 2, N. 47, Art\#455, Nov4, 2008.

Freqüentemente o rim direito é o mais acometido devido migração através da parede duodenal, porém, existe a possibilidade de se encontrar os parasitos em localização ectópica $[2,8,9,11]$.

A infecção conhecida como dioctofimose apresenta-se na maioria dos casos assintomática, podendo ocorrer sinais clínicos como cólica renal, disúria, hematúria, perda de peso acentuada, inquietação, dor lombar e eventualmente dor abdominal e peritonite, sendo feito o diagnóstico através do exame clínico, urinálise, radiografia, ultra-sonografia e achados acidentais em cirurgias e necropsias $[1,3,6]$.

No exame ultra-sonográfico normal do rim, a medula renal é menos ecogênica, seguida pelo córtex renal. A ecogenicidade do córtex renal é semelhante ou ligeiramente menor que a do parênquima de um fígado normal, e bem menor que a do parênquima esplênico. A gordura perirrenal que circunda o rim tem ecogenicidade similar ou maior que o proeminente complexo ecóico central do seio renal [7].

O diagnóstico ultra-sonográfico pode ser um método suficiente para sugerir a presença do parasito no parênquima renal, pela visualização de estruturas arredondadas, de aproximadamente $8 \mathrm{~mm}$ de diâmetro, com uma fina camada externa hiperecóica e centro hipoecóico [10].

\section{RELATO DE CASO}

Um animal, cão, sem raça definida (SRD) e procedente do Centro de Controle de Zoonoses (CCZ) do município de Cachoeiro de Itapemirim, estado do Espírito Santo, chegou ao Hospital Veterinário (HOVET) do Centro de Ciências Agrárias da Universidade Federal do Espírito Santo (CCA-UFES) com queixa de aumento de volume abdominal, este foi encaminhado ao Setor de Diagnóstico por Imagem para a realização de exame ultra-sonográfico. No exame realizado foi diagnosticado hiperemia de baço, aumento hepático e durante a varredura abdominal, na visualização do rim, o esquerdo revelou anatomia normal, porém o direito apresentou-se aumentado e com arquitetura 
Pereira, B.J., Holanda, C., Oliveira, D.C. et al. Diagnóstico ultra-sonográfico de dioctofimose em cão - relato de caso. PUBVET, Londrina, V. 2, N. 47, Art\#455, Nov4, 2008.

interna modificada, com várias estruturas tubulares e arredondadas apresentando-se com uma fina camada externa hiperecogênica e seu interior anecóico. Imagem sugestiva do parasito Dioctophyma renale. Após a ultrasonografia foi realizado urinálise com o intuito de pesquisar a presença de ovos, confirmando o diagnóstico.

\section{DISCUSSÃO E CONCLUSÃO}

A cadela chegou ao HOVET com a queixa de aumento de volume abdominal, com isso foi encaminhada para o Setor de Diagnóstico por Imagem para a realização do exame ultra-sonográfico. Diante do exame pode-se detectar a presença do parasito, e em concordância com a literatura consultada, o animal apresentava o rim direito parasitado $[2,8,9,11]$.

Esse relato destaca a importância do uso da ultra-sonografia, visto que o animal não seria submetido à urinálise caso não encontrasse o parasito. A imagem formada foi a descaracterização da região cortico-medular devido a presença do parasito. A imagem ultra-sonográfica do parasita no presente caso coincidiu com os relatos de Oliveira et al. Outra importância do diagnóstico ultra-sonográfico na dioctofimose é em relação ao número e sexo de parasitos no rim, pois caso haja apenas macho, não haverá a liberação de ovos pela urina, o que invalidaria a urinálise como método para definição do diagnóstico. 
Pereira, B.J., Holanda, C., Oliveira, D.C. et al. Diagnóstico ultra-sonográfico de dioctofimose em cão - relato de caso. PUBVET, Londrina, V. 2, N. 47, Art\#455, Nov4, 2008.

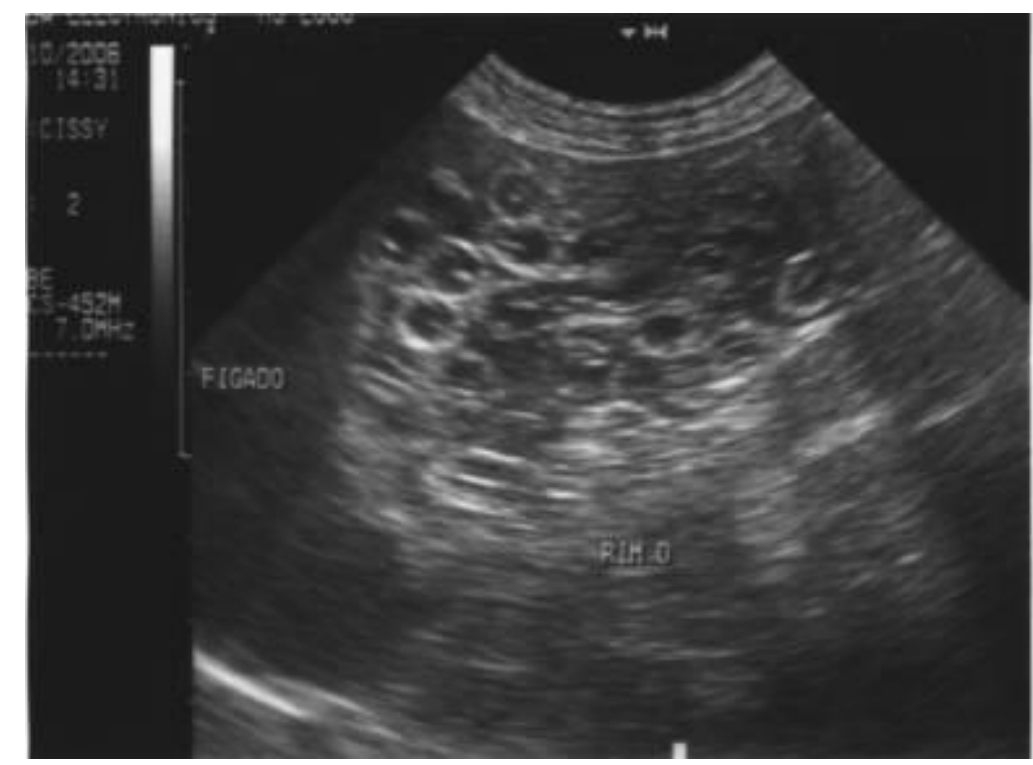

FIG 1.: Rim direito aumentado e com arquitetura interna modificada, com várias estruturas tubulares e arredondadas apresentando-se com uma fina camada externa hiperecogênica e seu interior anecóico.. Imagem sugestiva do parasito Dioctophyma renale.

\section{REFERÊNCIAS}

1. Acha, P. N.; Szyfres, B. Zoonoses and communicable diseases commom to man and animais. 3a Edição. Volume III. Parasitoses. Editora PAHO. Washington D.C., 2003. 544p

2. Barros, G. C. Dioctophyme renale (GOEZE, 1782) em cão no Estado do Espírito Santo. Arquivos da Escola de Veterinária. 23: 195-196.

3. Costa, P. R. dos Santos; Neto, N. M. A.; Oliveira, D. M. C.; Vasconcellos, R. S.; Menezes, F. M. Dioctofimose e leptospirose em uma cão - relato de caso. Clínica Veterinária. 51: 4850 .

4. Foreyt, W. J. Parasitologia veterinária: manual de referência. 5.ed. São Paulo: Roca, 40p.

5. Freitas, M.G. Helmintologia veterinária. 6.ed. Belo Horizonte: Precisa Editora Gráfica LTDA, 396p.

6. Júnior, L. C. M.; Maldonado, A. L. L.; Calheiros, D. F.; Paula, D. S. de; Ajzen, S. Dioctofimose em cão: relato de caso. Nosso clínico. 42: 14-18.

7. Nyland, T. G., Mattoon, J. S., Herrgesell, E. J., WisnerR, E. R. 2004. Trato urinário. In: Nyland, T. G.\& Mattoon, J. S. Ultra-som: Diagnóstico em pequenos animais. 2. ed. São Paulo: Roca, pp. 165. 
Pereira, B.J., Holanda, C., Oliveira, D.C. et al. Diagnóstico ultra-sonográfico de dioctofimose em cão - relato de caso. PUBVET, Londrina, V. 2, N. 47, Art\#455, Nov4, 2008.

8. Mattos Júnior, D. G.; Pinheiro, J. Dioctophyma renale(GOEZE, 1782) no tecido subcutâneo da região inguinal de um cão. Arquivo Brasileiro de Medicina Veterinária e Zootecnia. 46: 301-302.

9. Miranda, M. A.; Benigno, R. N. M.; Galvão G. R.; Oliveira S. A. L. Dioctophyma renale (GOEZE, 1782): Localização ectópica e alta intensidade parasitária em Canis familiares do Pará - Brasil. Arquivo Brasileiro de Medicina Veterinária e Zootecnia. 44: 151153.

10. Oliveira, L. L., Attallah, F. A., Santos, C. L., Wakofs, T. N., Rodrigues, M. C. D., Santos, A. E. O uso da ultrassonografia para o diagnóstico de Dioctophyma renale em cães Relato de caso. Revista Universitária Rural. 25: 323-324.

11. Osborne, C. A.; Stevenes, J. B.; Hanlon, G. F.; Rosin, E.; Bemrick, W. J. Dioctophyma renale in a dog. Journal of the American Veterinary Medical Association. 155: 605-620. 